\title{
Non-Suicidal Self-Injury and its Association with Identity Formation in India and Belgium:
}

\section{A Cross-Cultural Case-Control Study}

\begin{abstract}
Amarendra Gandhi
Leuven Biostatistics and Statistical Bioinformatics Centre (L-BioStat), Department of Public Health and Primary Care, KU Leuven, Leuven, Belgium
\end{abstract}

\section{Koen Luyckx}

Research Unit for School Psychology and Development in Context, KU Leuven, Leuven, Belgium

UNIBS, University of the Free State, Bloemfontein, South Africa

\author{
Alka Adhikari \\ Dhruv Parmar \\ Avinash Desousa \\ Nilesh Shah
}

Department of Psychiatry, Lokmanya Tilak Municipal Medical College, Mumbai, India

\section{Shubhada Maitra}

School of Social Work, Tata Institute of Social Sciences, Mumbai, India

\section{Laurence Claes}

Faculty of Psychology and Educational Sciences, KU Leuven, Leuven, Belgium Faculty of Medicine and Health Sciences, University of Antwerp, Antwerp, Belgium 


\section{Corresponding Author:}

Amarendra Gandhi

L-BioStat, Kapucijnenvoer 35 blok d - box 7001, 3000 Leuven, Belgium

(c/o Prof. Dr. Geert Molenberghs).

E-mail: Amarendra.Gandhi@kuleuven.be 


\begin{abstract}
The aim of the present study was to compare different features of Non-Suicidal Self-Injury (NSSI) in India and Belgium. We also explored whether the strength of the association between NSSI and disturbances in identity formation - a risk factor that can increase vulnerability to NSSI - was similar in young adults from India and Belgium. Data regarding NSSI and identity formation were collected from 182 young adults in India $(56 \%$ females, mean age $=21.5$ years, $S D=3.70$, range $=17-38$ years). The Belgian data used for matching were derived from four existing datasets. Of the 182 Indian cases, 138 cases could be matched with the Belgian sample on age, gender, and lifetime prevalence of NSSI. Lifetime prevalence of NSSI in the Indian sample was found to be around $21.4 \%$, with higher prevalence in females than in males. Comparison of features of NSSI in India and Belgium indicated that the age of onset of NSSI was higher in the Indian sample (around 17 years) than the Belgian sample (around 15 years). Additionally, self-bruising behavior was more commonly reported in India and scratching/cutting was more often reported in Belgium. Finally, the Belgian sample reported intra-personal functions of NSSI more often than the Indian sample. Moderation analysis indicated that the associations between NSSI and identity confusion/integration were stronger in the Belgian sample as compared to the Indian sample. Higher self-knowledge was protective against NSSI in both the Indian and the Belgian sample.
\end{abstract}

Keywords: Non-suicidal self-injury, cross-cultural comparison, identity formation, young adults, Belgium, India 


\section{Introduction}

Non-suicidal self-injury (NSSI) refers to the deliberate destruction of one's body tissue without an actual intention to die. The most common forms of NSSI include self-cutting, hitting, headbanging, and burning oneself (Nock, 2009). Engagement in even a single episode of NSSI has been demonstrated to be associated with a history of emotional, physical, or sexual abuse; suicidal behaviors (ideation, plan, or attempt); high levels of distress; and eating disorders symptoms (Whitlock, Eckenrode, \& Silverman, 2006). Much of the work on NSSI in the West indicates that, although the age of onset of NSSI peaks during mid-adolescence (around 15-16 years of age), risk of NSSI continues to be high even during young adulthood (Gandhi et al., 2017). Based on a review of observational studies, lifetime prevalence of NSSI in young adults have been found to be around 13\% (Swannell, Martin, Page, Hasking, \& St John, 2014). Consequently, NSSI is being increasingly identified as an important mental health concern even in young adults (Hamza \& Willoughby, 2016).

Existing research clearly indicates that NSSI is a global phenomenon that occurs in both Western and non-Western countries (Gholamrezaei, De Stefano, \& Heath, 2015; Swannell et al., 2014). However, the similarities and differences between the manifestation of NSSI in different countries remains unclear, as no research so far has compared NSSI between Western and nonWestern populations. Cross-cultural research may be instrumental not only in assessing the extent of the problem posed by NSSI in different countries but also in identifying culturally specific risk factors. To remedy this shortcoming, the present study compared different features of NSSI (e.g., age of onset, one-year frequency, and function) in India, a country with a predominantly Eastern culture, and Belgium, a country with a predominantly Western culture. Additionally, we investigated whether the association between NSSI and disturbances in identity formation - a risk factor that can increase vulnerability to NSSI - was similar in these two countries. 


\section{NSSI in India and Belgium}

A review of the existing literature on self-harming behavior in India indicates that, although a small number of studies have investigated behaviors similar to NSSI, inferring the prevalence of NSSI using these studies may be difficult, as they use definitions that may not allow for distinctions between different kinds of self-injurious behavior (e.g., attempted suicide and NSSI; Gandhi, Luyckx, Maitra, \& Claes, 2016). As far as we are aware, only two studies (Bhola, Manjula, Rajappa, \& Phillip, 2017; Kharsati \& Bhola, 2015) have researched NSSI in India (as defined in the present study) and they have produced somewhat conflicting results. The one-year prevalence of NSSI in India has been reported to vary between $31 \%$ and 34\%. However, as Bhola and colleagues (2017) point out, both studies used a very broad definition of NSSI. Therefore, the resulting one-year prevalence may be inflated. Neither study investigated the lifetime prevalence of NSSI. Apart from the prevalence of NSSI, evidence regarding gender differences also is conflicting, as one of the studies reported a lack of gender differences (Kharsati \& Bhola, 2015), whereas the other study reported a higher one-year prevalence of NSSI in females than in males (Bhola et al., 2017). Consistent with the international literature, the age of onset of NSSI in India has been reported to be around 15.9 years (Kharsati \& Bhola, 2015). Given the small number of studies and their inconsistent findings, further research is necessary to understand NSSI in India.

So far, the study by Kiekens and colleagues (2016) is the only one that has specifically investigated NSSI in young adults in Belgium. They reported a lifetime prevalence of NSSI of $8 \%$ and a one-year prevalence of 3\%. These authors also reported higher risk of NSSI in females. The age of onset of NSSI in Belgium has been shown to peak between 15 and 16 years (Gandhi et al., 2017). Based on the limited evidence, it appears that, apart from the age of onset, NSSI in India 
and Belgium may differ significantly on other characteristics. However, cross-cultural research is required to confirm these differences.

Apart from the epidemiological features, the functions of NSSI are also likely to be culturally influenced (Gholamrezaei et al., 2015). The functional approach suggests that NSSI is maintained through different reinforcement processes (Nock, 2009). These processes can be viewed as the functions of NSSI (Sadeh et al, 2014) and can be broadly categorized into intrapersonal and interpersonal; , each of which can be further classified into positive and negative reinforcement types. This yields four categories of functions of NSSI: 1 . Intrapersonal positive functions: to generate certain feelings/stimulation (e.g., to get into a trance or numb state); 2. Intrapersonal negative functions: to suppress aversive feeling or thoughts (e.g., to avoid or suppress negative feelings); 3. Interpersonal positive functions: to elicit support from others (e.g., to get attention from others); 4. Interpersonal negative functions: to escape undesirable social situations (e.g., to avoid doing chores or tasks; Nock, 2009).

A review of 35 studies of NSSI in non-Western countries suggests that individuals from these countries may engage in NSSI for more relational reasons as compared to their Western counterparts, while individuals from the West may engage in NSSI more for intrapersonal reasons (e.g., emotional regulation; Gholamrezaei et al., 2015). However, this assertion is not based on an actual comparison between Western and non-Western samples and, as such, it is not entirely supported by evidence from India. For example, although Kharsati and Bhola (2015) reported that individuals engaging in mild forms of NSSI more often reported its social functions, they also noted that those engaging in major NSSI behaviors were more likely to report intrapersonal functions, such as regulating negative emotions. 


\section{Disturbances in identity formation and NSSI}

Recent research suggests that, of the many intrapersonal and interpersonal issues that increase vulnerability to NSSI, disturbances in identity formation may be an important factor in both adolescents and young adults (Gandhi et al., 2017). Identity formation, according to Erikson (1968), is a process that begins during adolescence, when one's childhood identifications are no longer experienced as suitable, but a new identity has yet to be established. If individuals successfully develop an autonomous identity and a sense of sameness, identity synthesis is achieved. On the other hand, if individuals fail to develop an integrated sense of self, identity confusion ensues (Schwartz, 2001). Both cross-sectional (Claes, Luyckx, \& Bijttebier, 2014; Luyckx, Gandhi, Bijttebier, \& Claes, 2015) and longitudinal research (Gandhi et al., 2017), emerging mainly from Belgium, has demonstrated that whereas identity synthesis can reduce one's vulnerability to NSSI, persistent identity confusion can increase vulnerability to NSSI. However, using a cross-lagged analysis with two waves of data, Gandhi and colleagues (2017) were also able to demonstrate that engagement in NSSI may in turn hamper identity synthesis and increase identity confusion as well.

The process of identity formation is known to be influenced by cultural differences (Stevens, 2009) in at least two ways. First, unlike most Western cultures, which encourage the development of an autonomous self, most Eastern cultures emphasize the development of a relational self (Mascolo, Misra, \& Rapisardi, 2004). Consequently, it has been proposed that individuals from Eastern cultures mainly develop a sense of identity and self-knowledge based on input from families and the larger society, and less so on the basis of self-exploration and selfdiscovery (Cheng \& Berman, 2012). Second, identity distress due to lack of a consistent self does not necessarily lead to lower psychological adjustment in the East as much as it does in the West (Suh, 2002). That is, Indians, like other Eastern cultures, may not be as significantly affected by 
an inconsistent sense of self (Suh, 2002) as their Western counterparts. Although the exact reason for the tolerance of inconsistencies in self and identity in the Indian context is not clear, theoretical (Sinha, 2014) and empirical research (Berman et al., 2014; Rao et al., 2013) has indicated that such an ability may originate from the strongly multicultural and collectivistic nature of the Indian society. The acceptance of paradoxes and contradictions regarding self and others may be necessary in a culture in which the simultaneous existence of diverse beliefs, values, norms, and practices originating from varied religions, castes, and classes is a norm (Sinha, 2014). In sum, given the cultural differences in the process of identity formation and the differential effect of identity distress on general psychopathology, the extant research establishing the association between identity formation and NSSI in a Belgian context may not be sufficient. As such, cross-cultural confirmation of this association is necessary.

\section{The present study}

The present study had two main objectives. First, we compared epidemiological features of NSSI in young adults in India (predominantly representing an Eastern culture) and Belgium (predominantly representing a Western culture). More specifically, we compared: one-year prevalence of NSSI, lifetime prevalence of seven different forms of NSSI, age of onset of NSSI, body parts most often injured, and functions of NSSI between Indian and Belgian young adults. Based on the existing literature (Gholamrezaei et al., 2015), we expected the prevalence of the seven different forms of NSSI, body parts injured, and age of onset to be comparable between the Indian and the Belgian sample. Indian participants were expected to score higher on social or relational functions of NSSI, whereas the Belgian participants were expected to score higher on the self-related functions of NSSI. Second, we investigated if the strength of the association between identity formation and NSSI was influenced by nationality. Given the lack of existing 
research, this research question was considered as exploratory. Nonetheless, based on the work of Suh (2002), we expected the association between identity variables and NSSI to be stronger in Belgium than in India.

\section{Methods}

\section{Participants}

Indian data for the present study were collected at two institutes of higher education located in Mumbai, India, using convenience sampling. The first sample consisted of 120 (out of the total 150) first year bachelor of medicine and bachelor of surgery students (MBBS; $51.7 \%$ females) from a government medical college. Mean age was 19.7 years $(S D=2.16$, range $=17-28$ years $)$. The second sample consisted of 62 students ( $72.2 \%$ females) from a large government funded social sciences university. Mean age was 24.9 years $(S D=3.67$, range $=20-38$ years $)$. Therefore, the total sample from India consisted of 182 participants (56\% females). Mean age of the total sample was 21.5 years $(S D=3.70$, range $=17-38$ years $)$.

The Indian data were matched on age, gender, and presence/absence of lifetime NSSI with a sample of Belgian individuals. The Belgian data that were used for matching were derived from four existing datasets with information on age, gender, NSSI, and identity formation. These datasets were collected by the first, second, and/or the last authors in Belgium during the years 2012-2016. Whereas the Indian and the Belgian sample were matched exactly for gender and lifetime NSSI, a deviation of \pm 1 year was allowed around age. Of the 182 cases from India, 138 cases could be matched with the Belgian participants from the pooled datasets. The mean age of the participants in the reduced sample ( $n=138,57.2 \%$ females in both the samples) was 19.82 years $(S D=3.20)$ for the Indian sample and 20.28 years $(S D=2.90)$ for the Belgian sample. 


\section{Procedure}

Using a consent form, the first year MBBS students were informed about the purpose of the study, on and off campus mental health services available to them, and contact information of the researchers. One of the researchers was present throughout the data collection procedure to answer any questions from the students. The students were not compensated for participating in the study. The study was approved by the institutional ethics committee of the medical college. To optimize the data collection procedure in the social sciences institute, the survey was administered via the internet. The students of the social sciences university were invited to participate in the study via an e-mail that explained the purpose of the study, provided contact details of the researchers, and included the URL to the electronic survey form. The landing page of the data collection form provided detailed information on the study, instructions to complete the study survey, on and off campus mental health services available to students, and an electronic consent form. The researchers were available via e-mail to answer any questions the students had regarding the data collection procedure or the survey. Students were compensated with a Rs. 200 gift voucher for participating in the study. The study was approved by the institutional ethics committee of the Belgian university to which the first author belongs to (File number: S56676).

The data collection procedure for the four Belgian samples was similar to the procedure followed for the MBBS students in India. All the studies were approved by the ethics committee of the university of the first author.

\section{Measures}

Non-suicidal self-injury. The lifetime prevalence of NSSI was assessed by asking a single-item question: 'Have you ever engaged in self-injury without an intent to die?' (YES/NO 
answer format). If the participants answered the above question positively, they were further asked to indicate the lifetime prevalence of seven different forms of NSSI (scratching, carving, cutting, hitting or bruising, burning, pricking with a sharp object, and head banging) with YES/NO questions. The participants were also asked to indicate the age at which they started to engage in NSSI; if they were currently engaging in NSSI (YES/NO); and the body parts that were most often injured during NSSI. Finally, the participants were asked to indicate the degree to which they endorsed 18 functions of NSSI (for the list of the 18 functions, see Table 2; Claes \& Vandereycken, 2007) on a five-point Likert scale ranging from 1 ('not applicable') to 5 ('very applicable').

Identity formation. The Identity subscale of Erikson Psychological Stage Inventory (EPSI; Rosenthal, Gurney, \& Moore, 1981) is a 12-item scale that measures the extent to which the participants have a clear sense of who they are and in what they believe. Each item has to be scored on a 5-point Likert scale ranging from 1 ('Totally disagree') to 5 ('Totally agree'). Schwartz, Zamboanga, Wang, and Olthuis, (2009) suggested a bi-factor structure with 6 items measuring identity synthesis and 6 items measuring identity confusion. However, Bayesian confirmatory factor analysis indicated that a three-factor model fitted the data better than a twofactor model, for both the Indian and the Belgian samples. Therefore, the following three-factor solution for the EPSI scale was selected: (1) Confusion, (2) Integration, (3) Self-knowledge. For the details regarding the model fits and factor loadings, please refer to the online appendix. Cronbach's alpha for the three subscales for both India and Belgium samples and items for each sub-factor can be found in Table 3. The equivalence of the EPSI scale between Belgium and India was established using the approximate MI procedure (van de Schoot et al., 2013). The findings of these analysis can be accessed through the online supplement accompanying this manuscript. 


\section{Data Analysis}

Cross cultural comparison of NSSI. Cross-cultural differences in the one-year prevalence of NSSI, seven different forms of NSSI, and the body parts involved in self-harm were investigated using the $\chi^{2}$ test of differences. The Mann-Whitney U test was used to investigate group differences in the 18 functions of NSSI. In order to control for the inflation in Type 1 error due to multiple testing, the $p$-values were adjusted using the False Discovery Rate technique ([FDR]; 10\%; Benjamini \& Hochberg, 1995). Next, we used the discrete event history analysis method described by Gandhi and colleagues (2017) and by Singer, Willet, and Willet (2009) to investigate if the distribution of the age of onset of NSSI significantly differed between the Indian and the Belgian samples.

Moderation analysis. Finally, to investigate if the strength of the association between the identity variables and lifetime NSSI was influenced by nationality, three separate binomial logistic regression based moderation models (one for each of the three factors of the EPSI scale) were tested. In each model, lifetime NSSI was entered as the dependent variable; main effects of an identity variable and nationality, and their interaction were added as independent variables. To aid model interpretation, the predicted probability of NSSI for both nationalities, obtained from each model, was plotted against the relevant identity variable. Using the delta method (Xu \& Long, 2005, STATA, v14.1), we plotted 95\% confidence intervals around the differences between the predicted probability of NSSI between India and Belgium for various values of each of the identity variables. This procedure allowed us to investigate the values of identity variables that were associated with significant differences in the predicted probability of lifetime NSSI between India and Belgium. The predicted probability of lifetime NSSI is considered to be significantly 
different between the two countries, if the zero line does not pass through the area between upper and the lower limits of the confidence intervals.

\section{Results}

\section{Epidemiological features of NSSI in India}

The lifetime prevalence of NSSI in the total sample from India $(n=182)$ was $21.4 \%(n=39)$. Oneyear prevalence of NSSI was $4.3 \%(n=6)$. Females $(n=29)$ were more likely to engage in NSSI than males $\left(n=10 ; \chi^{2}=6.377, d f=1, p=.012\right)$. The $\chi^{2}$ tests indicated that there were no significant gender differences in the seven different kinds of NSSI. Additionally, there were no significant differences in the prevalence of NSSI between the students from the social sciences institute and the medical college $\left(\chi^{2}=.027, d f=1, p=.869\right)$.

\section{Cross-cultural comparison of NSSI}

One-year prevalence of NSSI did not differ significantly between the two countries $\left(\chi^{2}=.02\right.$, $d f=1, p=.897)$. The results of the $\chi^{2}$ test to examine associations between nationality and seven different forms of NSSI are shown in Table 1 (section $a$ ). In Table 2, scratching oneself till one bleeds and cutting oneself were more often seen in Belgium, whereas head banging was more often reported in the Indian sample. Section $b$ of Table 1 indicated that there was no significant association between nationality and the different body parts injured in NSSI.

Table 2 displays the findings of the Mann-Whitney U test to investigate the group differences in the 18 functions of NSSI. Table 2 shows that the distributions of item 3 (to avoid or suppress painful images or memories), 4 (to get into a trance or numb state), 9 (to avoid or suppress suicidal thoughts), and 16 (to avoid or suppress feelings of confusion/aimlessness) were 
significantly different from each other. The medians for the abovementioned items were higher in the Belgian sample than in the Indian sample.

The loglikelihood test and goodness of fit indices for models with various specifications of the baseline hazard function can be accessed in the online supplement accompanying this manuscript. The quadratic specification best fitted the data (fit better than the linear model and the lowest BIC) and therefore it was used as the baseline model. The coefficients of the quadratic model with nationality and its interaction with linear specification of age also can be accessed in the online appendix. The interaction of nationality and age was significant, indicating that the hazard of NSSI differed between the Indian and the Belgian sample. The predicted hazard function for the Indian and the Belgian samples (obtained from the model shown in Table 4 of the online supplement; the plot can be accessed in the online supplement accompanying this manuscript) was plotted against age. The plot indicated that the age of onset of NSSI peaked between the ages of 16-18 years (peak at 17) in the Indian sample, whereas in the Belgian sample, the age of onset peaked between the ages of 14-16 years (peak at 15).

\section{Moderation analysis}

Table 4 shows the regression coefficients for the moderation models which tested the effect of nationality on the strength of association between NSSI and the three identity variables. The regression coefficient indicates that, whereas the association between identity confusion and integration and NSSI was moderated by nationality, the association between self-knowledge and NSSI was not. The predicted probability plots for lifetime NSSI obtained from the three models are displayed in Figure 1. The first plot (Panel A1) shows that confusion positively predicted the probability of NSSI. Overall, the strength of the association between confusion and the probability of lifetime NSSI appeared to be relatively weak in the Indian sample. In contrast to the 
Indian sample, the association between confusion and NSSI was much stronger in the Belgian one. The probability of lifetime NSSI increased almost exponentially to more than .80 as the degree of identity confusion achieved its maximum score. The second plot (Panel B1) indicated that the association between integration and the probability of lifetime NSSI was negative. Difference in the predicted probability of NSSI between the two countries was significant only for very low values of integration $(\leq 1.5$; see Panel B2). Even with the low values of the integration scores, the predicted probability of lifetime NSSI was significantly higher in the Belgian as compared to the Indian sample. Finally, the third plot (Panel C1) shows that there was a negative association between self-knowledge and the probability of lifetime NSSI in both countries. Although the aforementioned association was stronger in Belgium than in India, this difference was not statistically significant (see Panel C2).

\section{Discussion}

The present study had two main objective: first, to compare epidemiological features of NSSI between two culturally distinct countries, India and Belgium; and second, to investigate whether the strength of the association between identity formation and NSSI was influenced by nationality. Our findings suggest that the lifetime prevalence of NSSI in young adults may be higher in India than in Belgium. In addition to these potential differences in prevalence, NSSI may also differ phenomenologically between the two countries. Finally, it appears as though the strength of the association between identity confusion and lifetime NSSI was the one that differed the most between India and Belgium. Each of these findings are discussed below.

First, although we did not directly compare the lifetime prevalence of NSSI between India and Belgium, the findings of the current study indicate that the lifetime prevalence and the oneyear prevalence of NSSI in Indian young adults was around $21 \%$ and $4 \%$, respectively. Therefore, 
the lifetime prevalence and one-year prevalence of NSSI may be higher in India than in Belgium (Kiekens et al., 2016). Note that the one-year prevalence of NSSI in the Indian sample in this study was almost $27 \%$ to $30 \%$ lower than that reported by earlier studies conducted by Kharsati and Bhola (2014) and Bhola and colleagues (2017). Although the difference in the one-year prevalence of NSSI may be explained by the difference in measurement methods (single YES/NO question vs. checklist), future studies with larger samples and with a research design combining both survey and interview techniques may be necessary to better understand NSSI in India. Consistent with the international literature (Bresin \& Schoenleber, 2015), we also found that females had a higher lifetime prevalence of NSSI than males in both countries.

Second, contrary to our expectations, cross-cultural comparison of NSSI between India and Belgium suggested several phenomenological differences. Whereas head banging was more often reported by Indian young adults, their Belgian counterparts reported more cutting and scratching behaviors. Based on these results and on the findings of the previous studies (Kharsati \& Bhola, 2014), it appears that Indian young adults may engage more often in self-bruising behaviors to harm themselves. Although the reason for favoring self-bruising over cutting is not clear, we conjecture that behaviors like cutting and subsequent wound care may require considerable privacy, which may not be easily available to urban Indians who live in close proximity with their families in relatively small residential spaces (Laungani, 2007). We observed that the Indian and the Belgian participants did not differ significantly from each other in regard to the most commonly injured body parts.

Our hypothesis regarding the differences between Belgium and India in the functions of NSSI were at least partially supported by our findings. As expected, Belgian participants engaged in NSSI for more intrapersonal reasons as compared to the Indian participants. The differential reporting of functions of NSSI may be due to the different cultural contexts of the two countries 
(Gholamrezaei et al., 2015). Given that Belgian culture is predominantly individualistic

(Goossens \& Luyckx, 2006), preponderance of self-related functions in Belgian young adults is not surprising. Additionally, given the interdependent and collectivistic nature of the Indian society (Laungani, 2007), social functions were expected to be more often reported by Indian participants. However, contrary to our expectations, the medians of interpersonal functions did not significantly differ between the two nationalities. Although it is not clear why the countries in our sample did not differ on social functions, the possibility of a non-significant result due to higher type 2 error resulting from a small sample size cannot be ruled out (Button et al., 2013).

In line with research emerging from Belgium (Claes et al., 2014; Luyckx, et al., 2015), the overall findings indicated that increased identity confusion also increased vulnerability to lifetime NSSI. However, as hypothesized, the strength of the association between identity confusion and lifetime NSSI differed significantly between India and Belgium. Low confusion was more protective against NSSI and high confusion was associated with higher vulnerability to NSSI in the Belgian sample than in the Indian sample. The moderation effect of culture on the association between identity confusion and NSSI may be an outcome of differences in how concepts like self and identity develop and operate in India and Belgium. As previously noted, an inconsistent self which lacks integration may be more acceptable in a non-Western context than in a Western country such as Belgium (Suh, 2002). On the other hand, identity integration and selfknowledge appeared to reduce one's vulnerability to NSSI. Findings of the moderation analysis indicated that the association of integration with lifetime NSSI significantly differed between the two cultures. However, a more fine-grained analysis using confidence intervals clarified that the predicted probability of lifetime NSSI was significantly higher in Belgium than in India only when there was a substantial lack of integration. 
If replicated in further longitudinal studies, the findings of the current study may have important clinical implications. First, the current study supports the need to address identity issues in the management of NSSI (Gandhi et al., 2017). Second, although increasing identity synthesis by improving integration and self-knowledge may be protective against NSSI in both Western and non-Western individuals, the way of achieving this goal may depend on culture. For example, given that individuals in the West often rely on self-exploration to consolidate their identity (Cheng \& Berman, 2012), introspective strategies to promote identity consolidation may work better in a Western context (Wilson \& Dunn, 2004). On the other hand, the interdependent nature of self and identity development observed in India may necessitate the use of relational approaches (e.g., seeing oneself through the eyes of others; Wilson \& Dunn, 2004) to help individuals from these countries to develop a better understanding of their identities.

The findings of the current study should be interpreted in the context of important limitations. First, the data were collected from a specific population (university students) using convenience sampling. Consequently, our findings may not be representative of the Indian or Belgian populations. Second, because of the cross-sectional design of our study, no claims regarding causal relations between identity formation and NSSI can be made. Cross-cultural longitudinal research is required to understand directionality of effects between these variables. Third, a review of international studies clearly indicates that NSSI often occurs in adolescence. Therefore, cross-cultural research with adolescent samples is needed. Fourth, in the present study, by matching the Belgian and the Indian samples on age and gender, we were able to control for the confounding effects of these variables. However, other factors, such as socioeconomic variables, may also influence the prevalence of NSSI (Gholamrezaei et al., 2015) and further research may benefit from considering these variables as controls. In the present study, we were unable to control for any additional sociodemographic variables as we did not have the necessary 
information in the Belgian samples. Fifth, extant research has demonstrated that both NSSI and identity formation may differ even between Western countries with similar cultural values (Plener, Libal, Keller, Fegert, \& Muehlenkamp, 2009). Large, multinational, multisite explorations of NSSI and associated risk and protective factors are needed to allow more systematic comparison of regional and group variation.

\section{Conclusion}

By using matched-samples and a robust analytical strategy, the present study suggested potential differences between Belgium and India in the prevalence, forms, and risk factors for NSSI . However, further research to establish the cross-cultural validity of NSSI and develop a more locally grounded understanding of forms of self-injury. Underlying key concepts like self and injury may have significantly different meanings in countries like India (Paranjpe, 2000;

Whitman, 2007). As well, the presence of many rituals that routinely involve mild to severe forms of self-injury may influence the way NSSI is expressed and perceived within the Indian context (see for example, Chittoria et al., 2014). Understanding the influence of cultural context on NSSI and on the development of may help us to further clarify the association between these constructs from an Indian perspective. Further research is also necessary to develop targeted interventions that are tailored to the psychosocial needs and cultural norms of the population being treated. 


\section{References}

Benjamini, Y., \& Hochberg, Y. (1995). Controlling the false discovery rate: a practical and powerful approach to multiple testing. Journal of the Royal Statistical Society. Series B (Methodological), 57, 289-300.

Berman, S. L., Ratner, K., Cheng, M., Li, S., Jhingon, G., \& Sukumaran, N. (2014). Identity distress during the era of globalization: A cross-national comparative study of India, China, and the United States. Identity, 14, 286-296.

Bhola, P., Manjula, M., Rajappa, V., \& Phillip, M. (2017). Predictors of non-suicidal and suicidal self-injurious behaviours, among adolescents and young adults in urban India. Asian Journal of Psychiatry, 29, 123-128.

Bresin, K., \& Schoenleber, M. (2015). Gender differences in the prevalence of nonsuicidal selfinjury: A meta-analysis. Clinical Psychology Review, 38, 55-64.

Button, K. S., Ioannidis, J. P., Mokrysz, C., Nosek, B. A., Flint, J., Robinson, E. S., \& Munafò, M. R. (2013). Power failure: why small sample size undermines the reliability of neuroscience. Nature Reviews Neuroscience, 14, 365-376.

Cheng, M., \& Berman, S. L. (2012). Globalization and identity development: A Chinese perspective. In S. J. Schwartz (Ed.), Identity around the world: New directions for child and adolescent development (pp. 103-121). New Jersey: Wiley.

Chittoria, R. K., Mohapatra, D. P., Friji, M. T., Kumar, S. D., Asokan, A., \& Pandey, S. (2014). Camphor burns of the palm and non-suicidal self-injury: An uncommonly reported, but socially relevant issue. Indian Journal of Plastic Surgery, 47, 252-255.

Claes, L., Luyckx, K., \& Bijttebier, P. (2014). Non-suicidal self-injury in adolescents: Prevalence and associations with identity formation above and beyond depression. Personality and Individual Differences, 61, 101-104. 
Claes, L., \& Vandereycken, W. (2007). The self-injury questionnaire-treatment related (SIQTR): Construction, reliability, and validity in a sample of female eating disorder patients. In P. M. Goldfarb (Ed.), Psychological tests and testing research trends (pp. 111-139). New York: Nova Science.

Erikson, E. (1968). Youth: Identity and crisis. New York: WW Norton \& Company.

Gandhi, A., Luyckx, K., Maitra, S., \& Claes, L. (2016). Non-suicidal self-injury and other selfdirected violent behaviors in India: A review of definitions and research. Asian journal of Psychiatry, 22, 196-201.

Gandhi, A., Luyckx, K., Maitra, S., Kiekens, G., Verschueren, M., \& Claes, L. (2017).

Directionality of effects between non-suicidal self-injury and identity formation: A prospective study in adolescents. Personality and Individual Differences, 109, 124-129.

Gelman, A., \& Carlin, J. (2014). Beyond power calculations: assessing type S (sign) and type M (magnitude) errors. Perspectives on Psychological Science, 9, 641-651.

Gholamrezaei, M., De Stefano, J., \& Heath, N. L. (2015). Nonsuicidal self-injury across cultures and ethnic and racial minorities: A review. International Journal of Psychology, 52, 316326.

Goossens, L., \& Luyckx, K. (2006). Belgium. In, J.J. Arnett (Ed), International encyclopedia of adolescence (pp. 64-76). New York: Taylor \& Francis.

Jha, S. D., \& Singh, K. (2011). An analysis of individualism-collectivism across Northern India. Journal of the Indian Academy of Applied Psychology, 37, 149-156.

Kharsati, N., \& Bhola, P. (2015). Patterns of non-suicidal self-injurious behaviours among college students in India. International Journal of Social Psychiatry, 61, 39-49. 
Kiekens, G., Claes, L., Demyttenaere, K., Auerbach, R. P., Green, J. G., Kessler, R. C., ... \& Bruffaerts, R. (2016). Lifetime and 12-month nonsuicidal self-injury and academic performance in college freshmen. Suicide and Life-Threatening Behavior, 46, 563-576.

Laungani, P. D. (2007). Understanding cross-cultural psychology: Eastern and western perspectives. California: Sage.

Luyckx, K., Gandhi, A., Bijttebier, P., \& Claes, L. (2015). Non-suicidal self-injury in high school students: Associations with identity processes and statuses. Journal of Adolescence, 41, $76-85$

Mascolo, M. F., Misra, G., \& Rapisardi, C. (2004). Individual and relational conceptions of self in India and the United States. New directions for child and adolescent development, 2004, 9-26.

Nock, M. K. (2009). Why do people hurt themselves? New insights into the nature and functions of self-injury. Current Directions in Psychological Science, 18, 78-83.

Paranjpe, A. C. (2001). Self and identity in modern psychology and Indian thought. New York: Plenum.

Plener, P. L., Libal, G., Keller, F., Fegert, J. M., \& Muehlenkamp, J. J. (2009). An international comparison of adolescent non-suicidal self-injury (NSSI) and suicide attempts: Germany and the USA. Psychological Medicine, 39, 1549-1558.

Rao, M. A., Berry, R., Gonsalves, A., Hastak, Y., Shah, M., \& Roeser, R. W. (2013). Globalization and the identity remix among urban adolescents in India. Journal of Research on Adolescence, 23, 9-24.

Rosenthal, D. A., Gurney, R. M., \& Moore, S. M. (1981). From trust on intimacy: A new inventory for examining Erikson's stages of psychosocial development. Journal of Youth and Adolescence, 10, 525-537. 
Sadeh, N., Londahl-Shaller, E. A., Piatigorsky, A., Fordwood, S., Stuart, B. K., McNiel, D. E., ... \& Yaeger, A. M. (2014). Functions of non-suicidal self-injury in adolescents and young adults with Borderline Personality Disorder symptoms. Psychiatry Research, 216, 217 222.

Schwartz, S. J. (2001). The evolution of Eriksonian and, neo-Eriksonian identity theory and research: A review and integration. Identity, 1, 7-58.

Schwartz, S. J., Zamboanga, B. L., Wang, W., \& Olthuis, J. V. (2009). Measuring identity from an Eriksonian perspective: Two sides of the same coin?. Journal of Personality Assessment, 91, 143-154.

Singer, J. D., Willett, J. B., \& Willett, J. B. (2003). Applied longitudinal data analysis: Modeling change and event occurrence. New York: Oxford university press.

Sinha, J. B. P. (2014). Psycho-Social Analysis of the Indian Mindset, Springer-India.

Stevens, R. (2008). Erik H. Erikson: Explorer of identity and the life cycle. Palgrave Macmillan: Vancouver.

Suh, E. M. (2002). Culture, identity consistency, and subjective well-being. Journal of Personality and Social Psychology, 83, 1378-1391.

Swannell, S.V., Martin, G.E., Page, A., Hasking, P. and St John, N.J., 2014. Prevalence of nonsuicidal self-injury in nonclinical samples: Systematic review, meta-analysis and metaregression. Suicide and Life Threatening Behavior, 44, 273-303.

Van De Schoot, R., Kluytmans, A., Tummers, L., Lugtig, P., Hox, J., \& Muthén, B. (2013). Facing off with Scylla and Charybdis: a comparison of scalar, partial, and the novel possibility of approximate measurement invariance. Frontiers in Psychology, 4, 1-15.

Whitlock, J., Eckenrode, J., \& Silverman, D. (2006). Self-injurious behaviors in a college population. Pediatrics, 117, 1939-1948. 
Whitman, S. M. (2007). Pain and suffering as viewed by the Hindu religion. The Journal of Pain, $8,607-613$.

Wilson, T. D., \& Dunn, E. W. (2004). Self-knowledge: Its limits, value, and potential for improvement. Annual Review of Psychology, 55, 493-519.

Xu, J., \& Long, J. S. (2005). Confidence intervals for predicted outcomes in regression models for categorical outcomes. Stata Journal, 5, 537-550 


\section{Biographical Notes}

Amarendra Gandhi, PhD, is a Postdoctoral Researcher at the Faculty of Psychology and Educational Sciences, KU Leuven. His research focuses on identity formation and non-suicidal self-injury in adolescents and young adults. His secondary research interests include cross-cultural and methodological research. [E-mail: Amarendra.Gandhi@kuleuven.be]

Koen Luyckx, PhD, is an Assistant Research Professor at the Research Unit for School Psychology and Development in Context at KU Leuven, Leuven, Belgium, and a research fellow at UNIBS, University of the Free State, Bloemfontein, South Africa. His research interests are identity development, psychopathology, and adaptation to chronic illness.

Alka Adhikari, MD, is Specialist Medical Officer in the Department of Psychiatry at Lokmanya Tilak Municipal Medical College and General Hospital, Sion, Mumbai. Her published work focuses on mental health services research, conceptual and methodological issues with adult populations, risk behavior, and biological aspects of psychiatric illnesses.

Dhruv Parmar, MBBS, is currently a second year student in the Diploma in Psychological Medicine, Resident Doctor in the Department of Psychiatry at Lokmanya Tilak Municipal Medical College and General Hospital, Sion Mumbai, India. Dr. Parmar's research pertains to mental health services. He is interested in the fields of digital psychology, sexual medicine, general psychiatry, deaddiction psychiatry and human behavior. His published work focuses on unique cases in psychiatry and studies in the field of digital psychology and sexual medicine. Has also presented a symposium in the field of E-psychiatry and the future of clinical practice.

Avinash Desousa, MD, DPM, MS, PsyD, is the Founder Trustee and Consultant Psychiatrist at Desousa Foundation. He is a Research Associate at the Department of Psychiatry, Lokmanya Tilak Municipal Medical College, Mumbai. His published work focuses on schizophrenia, child and adolescent psychiatric problems, ECT, and behavioral addictions.

Nilesh Shah, MD, DPM, DNB, is Professor and Head of the Department of Psychiatry at Lokmanya Tilak Municipal Medical College, Mumbai. He has over 35 years of clinical experience in psychiatry. He has over 150 publications and his main areas of interest include child psychiatry, ECT, and schizophrenia.

Shubhada Maitra, PhD, is Professor and Dean of the School of Social Work, Tata Institute of Social Sciences (TISS), Mumbai. She is the Faculty-in-Charge of two field action projects of TISS: Muskaan, the child and adolescent guidance centre of TISS, and Tarasha, which works towards recovery and reintegration of women living with mental illness. Her published work focuses on mental health, gender, sexuality and marital/couple relationships.

Laurence Claes, PhD, is a Full Professor at the Faculty of Psychology and Educational Sciences, KU Leuven, Leuven, Belgium and the Faculty of Medicine and Health Sciences, University of Antwerp, Antwerp, Belgium. Her research focuses on the associations between personality and psychopathology (eating disorders, non-suicidal self-injury, and pathological buying). 
Table 1. Nationality-based differences in the seven different forms of NSSI and the body parts involved in self-injury. Significant results are highlighted

a. Seven different forms of NSSI

\begin{tabular}{lllll}
\hline & Indian & Belgium & Chi-square & $p$-value \\
& $(n=29)$ & $(n=29)$ & $\left(d f^{+}=1\right)$ & \\
\hline Scratching & 4 & 12 & 5.524 & .019 \\
Carving & 10 & 11 & .075 & .785 \\
Cutting & 1 & 12 & 11.997 & .001 \\
Hitting or bruising & 9 & 16 & 3.445 & .063 \\
Burning & 1 & 5 & 2.974 & .085 \\
Pricking with sharp objects & 6 & 6 & .000 & 1.00 \\
Head banging & 17 & 8 & 5.695 & .017
\end{tabular}

b. Body-parts injured in self-injury

\begin{tabular}{|c|c|c|}
\hline Indian & Belgium & Chi-square \\
\hline$(n=29)$ & $(n=22)$ & $\left(d f^{\dagger}=1\right)$ \\
\hline
\end{tabular}

Head and neck

13

5

2.675

.102

Arms, hands, fingers, and nails 17

15

.110

.741

Torso, belly, and buttocks

4

6

1.248

.264

Legs, feet, and toes

2

4

1.535

.215

Breasts and genitals

0

0

NA

NA

$+d f=$ degrees of freedom 
Table 2. Mann-Whitney U test to investigate group differences in the 18 functions of NSSI.

Significant results are highlighted

\begin{tabular}{|c|c|c|c|c|c|c|c|}
\hline \multirow{2}{*}{$\begin{array}{l}\text { Sr } \\
\text { No }\end{array}$} & \multirow[b]{2}{*}{ Functions of NSSI } & \multicolumn{2}{|c|}{ Median } & \multirow[b]{2}{*}{$U$} & \multirow[b]{2}{*}{$p$} & \multirow{2}{*}{$\begin{array}{c}10 \% \\
\text { FDR } \\
\text { corrected } \\
\text { cut-off } \\
\text { for } p- \\
\text { value }^{1}\end{array}$} & \multirow[b]{2}{*}{$r$} \\
\hline & & $\begin{array}{l}\text { India } \\
n=29\end{array}$ & $\begin{array}{c}\text { Belgium } \\
n=26\end{array}$ & & & & \\
\hline 1 & To obtain a feeling of pleasure & 1 & 1 & 320 & .782 & .089 & -.05 \\
\hline 2 & To avoid or suppress negative feelings & 3 & 4 & 217.5 & .029 & .028 & -.30 \\
\hline 3 & To avoid or suppress painful images or memories & 1 & 3 & 193 & .006 & .011 & -.38 \\
\hline 4 & To get into a trance or numb state & 1 & 2 & 226 & .020 & .022 & -.32 \\
\hline 5 & To get attention from others & 1 & 1 & 291.5 & .334 & .067 & -.14 \\
\hline 6 & To escape from a trance or numb state & 1 & 1 & 244.5 & .062 & .033 & -.26 \\
\hline 7 & To punish myself & 2 & 3 & 274.5 & .256 & .056 & -.16 \\
\hline 8 & To make myself unattractive & 2.5 & 1 & 304.5 & .191 & .050 & -.22 \\
\hline 9 & To avoid or suppress suicidal thoughts & 1 & 2.5 & 201.5 & .003 & .006 & -.41 \\
\hline 10 & To show myself how strong I am & 1 & 1 & 307.5 & .545 & .078 & -.10 \\
\hline 11 & To show others how strong I am & 1 & 1 & 329 & 1.00 & .094 & -.02 \\
\hline 12 & To avoid doing chores or tasks I don't want to do & 1 & 1 & 290 & .143 & .039 & -.20 \\
\hline 13 & To escape from doing school, work, or other activities & 1 & 1 & 318.5 & .649 & .083 & -.06 \\
\hline 14 & To avoid being with other people & 1 & 1 & 302.5 & .283 & .061 & -.13 \\
\hline 15 & To avoid or suppress inner feelings of emptiness & 1 & 3 & 260.5 & .155 & .044 & -.20 \\
\hline 16 & To avoid or suppress feelings of confusion/aimlessness & 1 & 3.5 & 204 & .012 & .017 & -.35 \\
\hline 17 & To define myself as a person & 1 & 1 & 328.5 & 1.00 & .100 & -.02 \\
\hline 18 & To provide myself a sense of identity or individuality & 1 & 1 & 302.5 & .387 & .072 & -.14 \\
\hline
\end{tabular}


Table 3. Sample items and internal consistency coefficients (Cronbach's alpha) of the three subscales of the EPSI for the Indian and Belgian samples.

\begin{tabular}{llcc}
\hline EPSI subscales & \multicolumn{1}{c}{ Sample item } & \multicolumn{2}{c}{ Cronbach's alpha } \\
\cline { 3 - 4 } & I don't really know who I am. & .73 & Belgium Sample \\
\hline Confusion & I've got it together & .59 & .79 \\
Integration & I know what kind of person I am & .77 & .56 \\
Self-knowledge & Ind & & \\
\hline
\end{tabular}


Table 4. Logistic regression models with lifetime NSSI as the outcome variable, the main effect of three factors of the EPSI scale, main effect of nationality, and the interaction of the main effects.

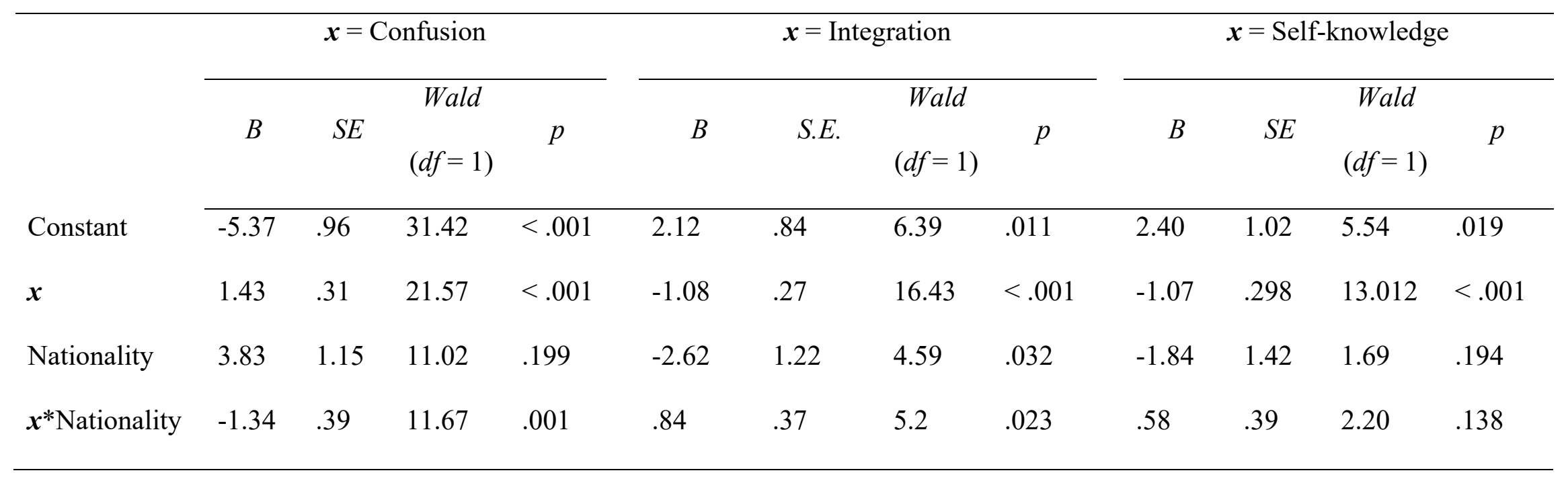


